\title{
On Zudilin-like rational approximations to $\zeta(5)$
}

\author{
Anier Soria Lorente \\ Department of Mathematics, University of Granma \\ Bayamo, Granma, Cuba \\ e-mail: asorial@udg.co.cu
}

Received: 2 May 2017

Accepted: 7 May 2018

\begin{abstract}
In this paper we obtain two Zudilin-Like recurrence relations of third order for $\zeta(5)$, after applying Zeilberger's algorithm of creative telescoping to some hypergeometric series. These recurrence relations do not supply diophantine approximations to $\zeta(5)$ that prove its irrationality, however it presents an algorithm for fast calculation of this constant. Moreover, we deduce a new continued fraction expansion for $\zeta(5)$ as a consequence.
\end{abstract}

Keywords: Riemann zeta function, Recurrence relation, Continued fraction expansion, Irrationality.

2010 Mathematics Subject Classification: Primary: 11B37, 30B70, 14G10, 11J72, 11M06; Secondary: 37B20, 11A55, 11J70, 11Y55, 11 Y65.

\section{Introduction}

The arithmetical properties of the Riemann zeta function at odd integer arguments

$$
\zeta(2 k+1) \equiv \sum_{n \geq 1} n^{-2 k-1}, \quad k \in \mathbb{N} \backslash\{0\},
$$

had fascinated a good number of mathematicians from the XVII century. In particular, Euler gave the following result for $\zeta(3)$

$$
\zeta(3)=\frac{\pi^{2}}{\log 2}+2 \int_{0}^{\pi / 2} x \log \sin x d x
$$

for more details, see [31]. In Addition, he exposed the following conjecture

$$
\zeta(2 k+1)=\frac{p}{q} \pi^{2 k+1},
$$


where $p$ and $q$ are integer numbers [33]. However, Euler's efforts to validate it were failed, and meanwhile the conjecture itself has been refuted [38]. Subsequent to the researches initiated by Euler, nothing was known on the arithmetical nature of the Riemann zeta function at odd arguments, until $2.00 \mathrm{pm}$ on a Thursday afternoon in June 1978, Roger Apéry surprised the mathematical community with a talk about the irrationality of $\zeta(3)$, see for instance $[7,33,34$, 41]. The aforesaid result was credited as Apéry's theorem, $\zeta(3) \notin \mathbb{Q},[7,8,9,10,11,12,15,18$, 21, 22, 29, 33, 34, 35, 36, 39, 40, 41].

From the result of Apéry, several seminaries were organized, in order to understand the aforesaid proof, so answering the questions about the arithmetical properties of the Riemann zeta function at odd integers. However, to this date it is not known if the $\zeta(5)$ is irrational or not, although many mathematicians conjecture that this aforesaid number is as much irrational like transcendental; some of the few results connected with $\zeta(5)$ appear in [30, 32, 42, 43, 44, 45]. Due to the importance conferred to the study of the arithmetical properties of $\zeta(2 k+1)$ for $k \in \mathbb{N}$, inside and outside of mathematics, many researchers, inspired by the ideas of Euler and of Apéry, have obtained some forms of representing to $\zeta(5)$. For example, in $[6,14]$ the authors showed the following relation connected to the golden ratio $\varphi=2^{-1}(\sqrt{5}+1)$ [37]

$$
\begin{aligned}
\zeta(5)=2^{-1} \sum_{k \geq 1} \frac{(-1)^{k+1}}{k^{5}\left(\begin{array}{c}
2 k \\
k
\end{array}\right)}+\frac{5}{4} \operatorname{Li}_{5}(\varphi)- & \frac{5}{4} \operatorname{Li}_{4}(\varphi) \\
& +2^{-1} \zeta(3) \log ^{2} \varphi-6^{-1} \zeta(2) \log ^{3} \varphi-48^{-1} \log ^{5} \varphi
\end{aligned}
$$

where

$$
\operatorname{Li}_{n}(z)=\sum_{k \geq 1} \frac{z^{k}}{k^{n}}
$$

is the polylogarithm of order $n$. In addition, in [13,27] appear

$$
\zeta(5)=2 \sum_{k \geq 1} \frac{(-1)^{k+1}}{k^{5}\left(\begin{array}{c}
2 k \\
k
\end{array}\right)}-\frac{5}{2} \sum_{k \geq 1} \frac{(-1)^{k+1}}{k^{3}\left(\begin{array}{c}
2 k \\
k
\end{array}\right)} \sum_{1 \leq j \leq k-1} \frac{1}{j^{2}},
$$

and in [26] it is deduced

$$
\begin{aligned}
& \zeta(5)=\frac{3}{16} \sum_{n \geq 1} \frac{(4 n-1)\left(16 n^{3}-8 n^{2}+4 n-1\right)}{(-1)^{n-1} n^{5}(2 n-1)\left(\begin{array}{c}
2 n \\
n
\end{array}\right)\left(\begin{array}{c}
3 n \\
n
\end{array}\right)} \\
& \quad+4^{-1} \sum_{n \geq 1} \frac{(-1)^{n}\left(56 n^{2}-32 n+5\right)}{n^{3}(2 n-1)^{2}\left(\begin{array}{c}
2 n \\
n
\end{array}\right)\left(\begin{array}{c}
3 n \\
n
\end{array}\right)} \sum_{1 \leq k \leq n-1} \frac{1}{k^{2}},
\end{aligned}
$$

and

$$
\begin{aligned}
\zeta(5)=\sum_{n \geq 1} \frac{(-1)^{n}\left(31 n^{2}-20 n+4\right)}{n^{7}\left(\begin{array}{c}
2 n \\
n
\end{array}\right)^{5}} \\
\quad+\sum_{n \geq 1} \frac{(-1)^{n}\left(205 n^{2}-160 n+32\right)}{n^{5}\left(\begin{array}{c}
2 n \\
n
\end{array}\right)^{5}}\left(\sum_{1 \leq k \leq n-1} \frac{1}{k^{2}}-\sum_{0 \leq k \leq n} \frac{1}{2(k+n)^{2}}\right),
\end{aligned}
$$


respectively. Another of the interesting results are the obtained for Zudilin, which, in [46] proved

$$
\begin{gathered}
F_{5, n}=(-1)^{n} n !^{4} \sum_{k \geq 1}\left(k+\frac{n}{2}\right) \frac{(1-k)_{n}(k+n+1)_{n}}{(k)_{n+1}^{6}} \\
=\int \cdots \int_{[0,1]^{5}} \frac{x_{1}^{n}\left(1-x_{1}\right)^{n} x_{2}^{n}\left(1-x_{2}\right)^{n} \cdots x_{5}^{n}\left(1-x_{5}\right)^{n}}{Q_{5}\left(x_{1}, x_{2}, \ldots, x_{5}\right)^{n+1}} d x_{1} d x_{2} \cdots d x_{5}=u_{n} \zeta(5)+w_{n} \zeta(3)-v_{n},
\end{gathered}
$$

with

$$
\begin{aligned}
Q_{5}\left(x_{1}, x_{2}, \ldots, x_{5}\right)=1-(1-(\cdots & \left.\left.\left(1-\left(1-x_{5}\right) x_{4}\right) \cdots\right) x_{2}\right) x_{1} \\
& =1-x_{1} Q_{4}\left(x_{2}, \ldots, x_{5}\right)=Q_{4}\left(x_{1}, \ldots, x_{4}\right)-x_{1} x_{2} \cdots x_{5}
\end{aligned}
$$

where $u_{n}, w_{n}, v_{n} \in \mathbb{Q}$ satisfy the following recurrence relation of third order

$$
(n+1)(n+2)^{5} b_{0}(n) y_{n+2}-b_{1}(n) u_{n+1}-b_{2}(n) u_{n}+2(2 n+1) n^{5} b_{0}(n+1) u_{n-1}=0
$$

with initial conditions

$$
\begin{aligned}
& u_{0}=2, \quad w_{0}=v_{0}=0 \\
& u_{1}=18, \quad w_{1}=66, \quad v_{1}=98 \\
& u_{2}=938, \quad w_{3}=\frac{6125}{2}, \quad v_{2}=\frac{74463}{16}
\end{aligned}
$$

where

$$
\begin{gathered}
b_{0}(n)=41218 n^{3}+48459 n^{2}+20010 n+2871, \\
b_{1}(n)=2(n+1)\left(3874492 n^{8}+33613836 n^{7}+123666762 n^{6}+250134420 n^{5}\right. \\
\left.+301587620 n^{4}+220011738 n^{3}+94372815 n^{2}+21917736 n+2131500\right), \\
b_{2}(n)=2\left(48802112 n^{9}+350188128 n^{8}+1080631646 n^{7}+1882848690 n^{6}\right. \\
+2045758212 n^{5}+1442754107 n^{4}+663248761 n^{3} \\
\left.+192486369 n^{2}+32136756 n+2360484\right) .
\end{gathered}
$$

Moreover, Zudilin in [45] using the very-well-poised hypergeometric series ${ }^{1}$ (1) as well as the result

$$
\tilde{F}_{5, n}=(-1)^{n+2} n !^{4} \sum_{k \geq 1}\left(k+\frac{n}{2}\right) \frac{(-k)_{n+1}(k+n)_{n+1}}{(k)_{n+1}^{6}},
$$

deduced the following recurrence relation of third order

$$
\begin{aligned}
& (n+1)^{6} \alpha_{0}(n) y_{n+1}+\alpha_{1}(n) y_{n}-4(2 n-1) \alpha_{2}(n) y_{n-1} \\
& -4(n-1)^{4}(2 n-1)(2 n-3) \alpha_{0}(n+1) y_{n-2}=0, \quad n \geq 2,
\end{aligned}
$$

\footnotetext{
${ }^{1}$ In the second section, we will give more details about this type of hypergeometric series.
} 
where

$$
\begin{aligned}
\alpha_{0}(n)= & 41218 n^{3}-48459 n^{2}+20010 n-2871, \\
\alpha_{1}(n)= & 2\left(48802112 n^{9}+89030880 n^{8}+36002654 n 7\right. \\
& -24317344 n^{6}-19538418 n^{5}+1311365 n^{4} \\
& \left.+3790503 n^{3}+460056 n^{2}-271701 n-60291\right), \\
\alpha_{2}(n)= & 3874492 n^{8}-2617900 n^{7}-3144314 n^{6} \\
& +2947148 n^{5}+647130 n^{4}-1182926 n^{3} \\
+ & 115771 n^{2}+170716 n-44541,
\end{aligned}
$$

which is satisfied by the numerators $p_{n, 5}$ and denominators $q_{n, 5}$ of the rational approximations to $\zeta(5)$ with the initial conditions

$$
\begin{aligned}
& p_{0,5}=0, \quad p_{1,5}=\frac{87}{2}, \quad p_{2,5}=-\frac{1190161}{64}, \\
& q_{0,5}=-1, \quad q_{1,5}=42, \quad q_{1,5}=-17934 .
\end{aligned}
$$

In addition, he verified that the sequence $r_{n, 5}=q_{n, 5} \zeta(5)-p_{n, 5}>0$ also satisfies the recurrence relation (2) and he checked that the same and the sequence of the denominators $q_{n, 5}$, satisfy the following limits

$$
\begin{aligned}
\lim _{n \rightarrow \infty} \frac{\log \left|r_{n, 5}\right|}{n} & =\log \left|\mu_{2}\right|=-1.08607936 \ldots, \\
\lim _{n \rightarrow \infty} \frac{\log \left|q_{n, 5}\right|}{n} & =\log \left|\mu_{3}\right|
\end{aligned}
$$

where

$$
\mu_{1}=-0.02001512 \ldots, \quad \mu_{2}=0.33753726 \ldots, \quad \mu_{3}=-2368.31752213 \ldots,
$$

are the roots of the characteristic polynomial $\mu^{3}+2368 \mu^{2}-752 \mu-16$ of recurrence relation (2). With these results, Zudilin presented an efficient and fast algorithm for the calculation of this constant $\zeta(5)$, since the sequence of rational approximations $p_{n, 5} / q_{n, 5}$ converge to $\zeta(5)$ with speed $\left|\mu_{2} / \mu_{3}\right|<1.42521964 \cdot 10^{-4}[33,45]$.

The aim of this paper is to present an efficient algorithm for fast calculation of $\zeta(5)$. As consequence, new Zudilin-like rational approximations to $\zeta(5)$ are deduced, as well as a new continued fraction expansion for this constant.

\section{Main results}

As it is known, the ordinary hypergeometric series $[17,20,23]$ at the variable $z$ is defined by

$$
{ }_{r} F_{s}\left(\begin{array}{c}
a_{1}, \ldots, a_{r} \\
b_{1}, \ldots, b_{s}
\end{array} \mid z\right) \equiv \sum_{k \geq 0} \frac{\left(a_{1}\right)_{k} \cdots\left(a_{r}\right)_{k}}{\left(b_{1}\right)_{k} \cdots\left(b_{s}\right)_{k}} \frac{z^{k}}{k !},
$$

where $(\cdot)_{k}$ denotes the Pochhammer symbol $[5,16]$, also called the shifted factorial, defined by 


$$
\begin{aligned}
& (z)_{k} \equiv \prod_{0 \leq j \leq k-1}(z+j), \quad k \geq 1, \\
& (z)_{0}=1, \quad(-z)_{k}=0, \quad \text { if } z<k,
\end{aligned}
$$

which in terms of the gamma function is given by

$$
(z)_{k}=\frac{\Gamma(z+k)}{\Gamma(z)}, \quad k=0,1,2, \ldots
$$

Here $\left\{a_{i}\right\}_{i=1}^{r}$ and $\left\{b_{j}\right\}_{j=1}^{s}$ are complex numbers subject to the condition that $b_{j} \neq-n$ with $n \in \mathbb{N} \backslash\{0\}$ for $j=1,2, \ldots, s$. In particular, the series

$$
{ }_{r+1} F_{r}\left(\begin{array}{c}
a_{0}, a_{1}, \ldots, a_{r} \\
b_{1}, \ldots, b_{r}
\end{array} \mid z\right)=\sum_{k \geq 0} \frac{\left(a_{0}\right)_{k} \cdots\left(a_{r}\right)_{k}}{\left(b_{1}\right)_{k} \cdots\left(b_{r}\right)_{k}} \frac{z^{k}}{k !},
$$

is called well-poised if the sequences $\left\{a_{i}\right\}_{i=0}^{r}$ and $\left\{b_{j}\right\}_{j=1}^{r}$ satisfy the following relations

$$
a_{0}+1=a_{1}+b_{1}=a_{2}+b_{2}=\cdots=a_{r}+b_{r} .
$$

Theorem 2.1. Let $n$ be an integer, with $n \geq 1$. Then, the following sequences

$$
\mathcal{R}_{n, 1}=(-1)^{n} n !^{4} \sum_{k \geq 1}(2 k+n+2) \frac{(-k)_{n}(k+n+2)_{n}}{(k+1)_{n+1}^{6}},
$$

and

$$
\mathcal{R}_{n, 2}=(-1)^{n} n !^{4} \sum_{k \geq 1}(2 k+n+2) \frac{(1-k)_{n-1}(k+n+3)_{n-1}}{(k+1)_{n+1}^{6}},
$$

are the very-well-poised hypergeometric series

$$
\frac{n !^{11}(3 n+2) !}{(2 n+1) !^{7}} F_{8}\left(\begin{array}{c|c}
3 n+2, \frac{3 n}{2}+2, n+1, \ldots, n+1 \\
\frac{3 n}{2}+1,2 n+2, \ldots, 2 n+2 & 1
\end{array}\right)
$$

and

$$
-\frac{(n-1) !(3 n+2) ! n !^{10}}{(2 n+2) !(2 n+1) !^{6}}{ }_{9} F_{8}\left(\begin{array}{c}
3 n+2, \frac{3 n}{2}+2, n, n+1, \ldots, n+1 \\
\frac{3 n}{2}+1,2 n+3,2 n+2, \ldots, 2 n+2
\end{array} \mid 1\right)
$$

respectively.

Proof. We prove only the second result, since both are similar. According to (3) we have

$$
\mathcal{R}_{n, 2}=(-1)^{n} n !^{4} \sum_{k \geq n}(2 k+n+2) \frac{(1-k)_{n-1}(k+n+3)_{n-1}}{(k+1)_{n+1}^{6}} .
$$


Consequently

$$
\mathcal{R}_{n, 2}=2(-1)^{n} n !^{4} \sum_{k \geq 0}\left(k+\frac{3}{2} n+1\right) \frac{(1-k-n)_{n-1}(k+2 n+3)_{n-1}}{(k+n+1)_{n+1}^{6}} .
$$

Then, having into account

$$
\begin{aligned}
& \left(k+\frac{3}{2} n+1\right)=\frac{2^{-1}(3 n+2)\left(\frac{3 n}{2}+2\right)_{k}}{\left(\frac{3 n}{2}+1\right)_{k}}, \\
& (-1)^{n-1}(1-k-n)_{n-1}=\frac{(n-1) !(n)_{k}}{k !}, \\
& (k+2 n+3)_{n-1}=\frac{(3 n+1) !(3 n+2)_{k}}{(2 n+2) !(2 n+3)_{k}},
\end{aligned}
$$

and

$$
(k+n+1)_{n+1}^{6}=\frac{(2 n+1) !^{6}(2 n+2)_{k}^{6}}{n !^{6}(n+1)_{k}^{6}} .
$$

We deduce

$$
\mathcal{R}_{n, 2}=-\frac{(n-1) !(3 n+2) ! n !^{10}}{(2 n+2) !(2 n+1) !^{6}} \sum_{k \geq 0} \frac{(3 n+2)_{k}\left(\frac{3 n}{2}+2\right)_{k}(n)_{k}(n+1)_{k}^{6}}{\left(\frac{3 n}{2}+1\right)_{k}(2 n+3)_{k}(2 n+2)_{k}^{6}},
$$

which coincides with (6). This finishes the proof, the detailed verification of (7)-(10) being left to the reader.

Indeed, the very-well-poised hypergeometric series (4) and (5) are $\mathbb{Q}$-linear forms in

$$
\{1, \zeta(3), \zeta(5)\}
$$

i. e.,

$$
\mathcal{R}_{n, 1}=\alpha_{n} \zeta(5)+\beta_{n} \zeta(3)-\gamma_{n} \quad \text { and } \quad \mathcal{R}_{n, 2}=\tilde{\alpha}_{n} \zeta(5)+\tilde{\beta}_{n} \zeta(3)-\tilde{\gamma}_{n}
$$

Next, we apply to the very-well-poised hypergeometric series (4) and (5), the so-called algorithm of creative telescoping due to W. Gosper and D. Zeilberger [1, 2, 3, 4, 25], from which are deduced the following results. In fact, this algorithm is implemented in different computer algebra systems, in particular, in Maple and Mathematica.

Proposition 2.2. The sequences $\left(\alpha_{n}\right)_{n \geq 1},\left(\beta_{n}\right)_{n \geq 1},\left(\gamma_{n}\right)_{n \geq 1}$ and $\left(\mathcal{R}_{n, 1}\right)_{n \geq 1}$ verify the following Zudilin-like recurrence relation

$$
\begin{aligned}
(n+2)(n+3)^{5} \eta_{n}^{(1)} y_{n+3}-2(n+2) \eta_{n}^{(2)} y_{n+2} & -2 \eta_{n}^{(3)} y_{n+1} \\
& +2(n+1)^{5}(2 n+3) \eta_{n}^{(4)} y_{n}=0, \quad n \geq 1,
\end{aligned}
$$

with initial conditions

$$
\begin{gathered}
\alpha_{1}=18, \quad \beta_{1}=66, \quad \gamma_{1}=98 \\
\alpha_{2}=938, \quad \beta_{2}=\frac{6125}{2}, \quad \gamma_{2}=\frac{74463}{16}, \\
\alpha_{3}=77202, \quad \beta_{3}=\frac{1524635}{6}, \quad \gamma_{3}=\frac{1498833983}{3888},
\end{gathered}
$$


where

$$
\begin{gathered}
\eta_{n}^{(1)}=41218 n^{3}+172113 n^{2}+240582 n+112558 \\
\eta_{n}^{(2)}=3874492 n^{8}+64609772 n^{7}+467449390 n^{6} \\
+1914997100 n^{5}+4854959850 n^{4}+7794497470 n^{3} \\
+7734655711 n^{2}+4336014520 n+1051310919 \\
\eta_{n}^{(3)}=48802112 n^{9}+789407136 n^{8}+5639012702 n^{7}+23351915204 n^{6} \\
+61795716198 n^{5}+108398618199 n^{4}+126080841295 n^{3} \\
+93794477946 n^{2}+40508900959 n+7741215265
\end{gathered}
$$

and

$$
\eta_{n}^{(4)}=41218 n^{3}+295767 n^{2}+708462 n+566471
$$

Proposition 2.3. The sequences $\left(\tilde{\alpha}_{n}\right)_{n \geq 1},\left(\tilde{\beta}_{n}\right)_{n \geq 1},\left(\tilde{\gamma}_{n}\right)_{n \geq 1}$ and $\left(\mathcal{R}_{n, 2}\right)_{n \geq 1}$ verify the following Zudilin-like recurrence relation

$$
\begin{aligned}
(n+3)^{3}(n+4)^{6} \tilde{\eta}_{n}^{(1)} y_{n+3}-2(n & +3)^{4} \tilde{\eta}_{n}^{(2)} y_{n+2}-2(n+2)^{3} \tilde{\eta}_{n}^{(3)} y_{n+1} \\
& +2 n(n+1)^{5}(n+2)^{2}(2 n+3) \tilde{\eta}_{n}^{(4)} y_{n}=0, \quad n \geq 1,
\end{aligned}
$$

with initial conditions

$$
\begin{gathered}
\tilde{\alpha}_{1}=8, \quad \tilde{\beta}_{1}=28, \quad \tilde{\gamma}_{1}=\frac{2685}{64}, \\
\tilde{\alpha}_{2}=222, \quad \tilde{\beta}_{2}=\frac{1455}{2}, \quad \tilde{\gamma}_{2}=\frac{12885155}{11664}, \\
\tilde{\alpha}_{3}=11340, \quad \tilde{\beta}_{3}=\frac{223895}{6}, \quad \tilde{\gamma}_{3}=\frac{56350012781}{995328},
\end{gathered}
$$

where

$$
\begin{gathered}
\tilde{\eta}_{n}^{(1)}=41218 n^{9}+761976 n^{8}+6198908 n^{7}+29116116 n^{6} \\
+86990832 n^{5}+171424173 n^{4}+222811091 n^{3}+184222071 n^{2} \\
+87944623 n+18475776, \\
\tilde{\eta}_{n}^{(2)}=3874492 n^{14}+135554862 n^{13}+2182452596 n^{12}+21427732081 n^{11} \\
+143302454897 n^{10}+690442234873 n^{9}+2471123718840 n^{8} \\
+6673220764546 n^{7}+13662032585953 n^{6}+21099764318756 n^{5} \\
+24196514657956 n^{4}+19978330639676 n^{3} \\
+11227117507832 n^{2}+3843841185536 n+604947087360,
\end{gathered}
$$




$$
\begin{aligned}
\tilde{\eta}_{n}^{(3)}=48802112 n^{15}+1780617600 n^{14}+30035105938 n^{13}+310655379860 n^{12} \\
+2203200295168 n^{11}+11348221528255 n^{10}+43853910213752 n^{9} \\
+129467584214041 n^{8}+294411248543850 n^{7}+515715215712131 n^{6} \\
+690243684548826 n^{5}+693281686236195 n^{4}+505903008137430 n^{3} \\
+253247361204846 n^{2}+77776973364636 n+11049992110080
\end{aligned}
$$

and

$$
\begin{aligned}
\tilde{\eta}_{n}^{(4)}=41218 n^{9}+1132938 n^{8}+13778564 n^{7}+ & 97306112 n^{6} \\
+ & 439728720 n^{5}+1318613641 n^{4}+2623833171 n^{3} \\
+ & 3340846686 n^{2}+2470028712 n+807986784 .
\end{aligned}
$$

Remark 2.4. Observe that the initial conditions (13) and (15) are justified by the following relations

$$
\begin{aligned}
& \mathcal{R}_{1,1}=18 \zeta(5)+66 \zeta(3)-98, \\
& \mathcal{R}_{2,1}=938 \zeta(5)+\frac{6125}{2} \zeta(3)-\frac{74463}{16}, \\
& \mathcal{R}_{3,1}=77202 \zeta(5)+\frac{1524635}{6} \zeta(3)-\frac{1498833983}{3888},
\end{aligned}
$$

and

$$
\begin{aligned}
& \mathcal{R}_{1,2}=8 \zeta(5)+28 \zeta(3)-\frac{2685}{64}, \\
& \mathcal{R}_{2,2}=222 \zeta(5)+\frac{1455}{2} \zeta(3)-\frac{12885155}{11664}, \\
& \mathcal{R}_{3,2}=11340 \zeta(5)+\frac{223895}{6} \zeta(3)-\frac{56350012781}{995328},
\end{aligned}
$$

which are easy to check using (4) and (5), respectively.

Evidently, from (11) we deduce that $r_{n}=q_{n} \zeta(5)-p_{n}$, where

$$
q_{n}=\alpha_{n} \tilde{\beta}_{n}-\tilde{\alpha}_{n} \beta_{n}, \quad p_{n}=\tilde{\beta}_{n} \gamma_{n}-\beta_{n} \tilde{\gamma}_{n} \quad \text { and } \quad r_{n}=\tilde{\beta}_{n} \mathcal{R}_{n, 1}-\beta_{n} \mathcal{R}_{n, 2} .
$$

As the characteristic equation of (12) and (14) is

$$
\lambda^{3}-188 \lambda^{2}-2368 \lambda+4=0,
$$

and its zeros are $t_{1}=0.00168896+7.10543 \times 10^{-15} i, t_{2}=-11.8505-7.10543 \times 10^{-15} i$ and $t_{3}=199.849-5.92119 \times 10^{-16} i$. Then, by from Poincaré's theorem [24, 28] we deduced that $\alpha_{n}=\mathcal{O}\left(\left|t_{3}\right|^{n}\right), \beta_{n}=\mathcal{O}\left(\left|t_{3}\right|^{n}\right), \gamma_{n}=\mathcal{O}\left(\left|t_{3}\right|^{n}\right)$ and $\mathcal{R}_{n, 1}=\mathcal{O}\left(\left|t_{1}\right|^{n}\right)$, respectively, as $n$ goes to infinity. Observe that the same behavior occurs for $\tilde{\alpha}_{n}=\mathcal{O}\left(\left|t_{3}\right|^{n}\right), \tilde{\beta}_{n}=\mathcal{O}\left(\left|t_{3}\right|^{n}\right), \tilde{\gamma}_{n}=\mathcal{O}\left(\left|t_{3}\right|^{n}\right)$ and $\mathcal{R}_{n, 2}=\mathcal{O}\left(\left|t_{1}\right|^{n}\right)$. Thus, from above results we follow to following conjecture.

Conjecture 2.1. Let $n$ be positive integer, with $n \geq 1$. Then, the sequences $\left(p_{n}\right)_{n \geq 1},\left(q_{n}\right)_{n \geq 1}$ and $\left(r_{n}\right)_{n \geq 1}$, have the following behavior $p_{n}=\mathcal{O}\left(\left|t_{2} t_{3}\right|^{n}\right), q_{n}=\mathcal{O}\left(\left|t_{2} t_{3}\right|^{n}\right)$ and $r_{n}=\mathcal{O}\left(\left|t_{1} t_{3}\right|^{n}\right)$, as $n$ goes to infinity. 
Evidently, the previous conjecture supplies an algorithm for fast calculation of the number $\zeta(5)$. Consequently, the rational approximations $p_{n} / q_{n}$ converge to $\zeta(5)$ with speed $1.42522 \times$ $10^{-4}$, which is showed in the following Table 1.

\begin{tabular}{|c|c|c|}
\hline$n$ & $p_{n} / q_{n}$ & $\left|\zeta(5)-p_{n} / q_{n}\right|$ \\
\hline 1 & $\frac{797}{768}$ & 0.0008327 \\
\hline 2 & $\frac{6095741}{5878656}$ & $9.685 \times 10^{-8}$ \\
\hline 3 & $\frac{13823722765}{13331423232}$ & $1.321 \times 10^{-11}$ \\
\hline 4 & $\frac{694059844981027}{669342528000000}$ & $1.836 \times 10^{-15}$ \\
\hline 5 & $\frac{116185685519039939851}{112048004253657600000}$ & $2.578 \times 10^{-19}$ \\
\hline 6 & $\frac{3796057669715104060275403}{3660869960212652812800000}$ & $3.638 \times 10^{-23}$ \\
\hline 7 & $\frac{1883364094989235447800132560011}{1816292490626633604983193600000}$ & $5.149 \times 10^{-27}$ \\
\hline 10 & $\vdots$ & $1.472 \times 10^{-38}$ \\
\hline 20 & $\vdots$ & $5.016 \times 10^{-77}$ \\
\hline 47 & $\vdots$ & $7.106 \times 10^{-181}$ \\
\hline 70 & $\vdots$ & $2.455 \times 10^{-269}$ \\
\hline
\end{tabular}

Table 1. Rational approximations to $\zeta(5)$

Let us recall some results about the continued fraction representation. We say that a number $\alpha$ can be written by a infinite irregular continued fraction expansion, if admits the following representation

$$
\alpha=a_{0}+\frac{b_{1} \mid}{\mid a_{1}}+\frac{b_{2} \mid}{\mid a_{2}}+\cdots+\frac{b_{n} \mid}{\mid a_{n}}+\cdots=a_{0}+\frac{b_{1}}{a_{1}+\frac{b_{2}}{a_{2}+\frac{b_{3}}{a_{3}+\ddots_{a_{n-1}}+\frac{b_{n}}{a_{n}+} \ddots_{\ddots}}}}
$$

Theorem 2.5. [19, p. 31] Let $\left(p_{n}\right)_{n \geq-1}$ and $\left(q_{n}\right)_{n \geq-1}$ be two sequences of numbers such that $q_{-1}=0, p_{-1}=q_{0}=1$ and $p_{n} q_{n-1}-p_{n-1} q_{n} \neq 0$ for $n=0,1,2, \ldots$ Then, there exists a unique irregular continued fraction

$$
a_{0}+\frac{b_{1} \mid}{\mid a_{1}}+\frac{b_{2} \mid}{\mid a_{2}}+\frac{b_{3} \mid}{\mid a_{3}}+\cdots+\frac{b_{n} \mid}{\mid a_{n}}+\cdots
$$


whose $n$-th numerator is $p_{n}$ and $n$-th denominator is $q_{n}$, for each $n \geq 0$. More precisely

$$
\begin{gathered}
a_{0}=p_{0}, \quad a_{1}=q_{1}, \quad b_{1}=p_{1}-p_{0} q_{1} \\
a_{n}=\frac{p_{n} q_{n-2}-p_{n-2} q_{n}}{p_{n-1} q_{n-2}-p_{n-2} q_{n-1}}, \quad b_{n}=\frac{p_{n-1} q_{n}-p_{n} q_{n-1}}{p_{n-1} q_{n-2}-p_{n-2} q_{n-1}}, \quad n=0,1,2, \ldots
\end{gathered}
$$

Theorem 2.6. [19, p. 31] Two irregular continued fractions

$$
a_{0}+\frac{b_{1} \mid}{\mid a_{1}}+\frac{b_{2} \mid}{\mid a_{2}}+\frac{b_{3} \mid}{\mid a_{3}}+\cdots+\frac{b_{n} \mid}{\mid a_{n}}+\cdots, \quad a_{0}^{\prime}+\frac{b_{1}^{\prime} \mid}{\mid a_{1}^{\prime}}+\frac{b_{2}^{\prime} \mid}{\mid a_{2}^{\prime}}+\frac{b_{3}^{\prime} \mid}{\mid a_{3}^{\prime}}+\cdots+\frac{b_{n}^{\prime} \mid}{\mid a_{n}^{\prime}}+\cdots
$$

are equivalent if and only if there exists a sequence of non-zero $\left(c_{n}\right)_{n \geq 0}$ with $c_{0}=1$ such that

$$
a_{n}^{\prime}=c_{n} a_{n}, \quad n=0,1,2, \ldots, \quad b_{n}^{\prime}=c_{n} c_{n-1} b_{n}, \quad n=1,2, \ldots
$$

Using the previous theorems we deduce the following results.

Theorem 2.7. The following irregular continued fraction expansion for $\zeta(5)$ is verify

$$
\begin{array}{r}
\zeta(5)=\frac{797 \mid}{\mid 768}+\frac{-37597440 \mid}{\mid-60957410}+\frac{4963010140935 \mid}{\mid-699335469} \\
+\frac{15299843303372544 \mid}{\mid-160388693712}+\frac{442065924497557800000 \mid}{\mid-19820970745081} \\
+\frac{2826977104806064592400532800 \mid}{\mid-1015388502751019592} \\
+\frac{161193705016034065874069140445355480 \mid}{\mid}+\cdots
\end{array}
$$

\section{Acknowledgements}

The author wishes to thank to ClaveMat project, financed by European Union, www.clavemat.com, and the University of Granma, where the paper was written.

\section{References}

[1] Abramov, S. A. (2002) Applicability of Zeilberger's algorithm to hypergeometric terms, In ISSAC'02: Proceedings of the 2002 International Symposium on Symbolic and Algebraic Computation, New York, 1-7.

[2] Abramov, S. A. \& Le, H. Q. (2002) A criterion for the applicability of Zeilberger's algorithm to rational functions, Discrete Math., 259, 1-17.

[3] Abramov, S. A. (2003) When does Zeilberger's algorithm succeed?, Appl. Math., 30, 424441, 2003. 
[4] Abramov, S. A., Carette, J. J., Geddes, K. O. \& Le, H.Q. (2004) Telescoping in the context of symbolic summation in Maple, J. of Symb. Comput., 30, 1303-1326.

[5] Abramowitz, M. \& Stegun, I. A. (1972) Handbook of Mathematical Functions with Formulas, Graphs, and Mathematical Tables, Dover, New York.

[6] Almkvist, G. \& Granville, A. (1999) Borwein and Bradley's Apéry-like formulae for $\zeta(4 n+3)$, Experiment. Math., 8, 197-203.

[7] Apéry, R. (1979) Irrationalité de $\zeta(2)$ et $\zeta(3)$, Astérisque, 61, 11-13.

[8] Arvesú, J. (2012) Orthogonal forms: A key tool for deducing Apéry's recurrence relation, J. Approx. Theory, accepted, 2012.

[9] Beukers, F. (1979) A note on the irrationality of $\zeta(2)$ and $\zeta(3)$, Bull. London Math. Soc., 11, 268-272.

[10] Beukers, F. (1980) Legendre polynomials in irrationality proofs, Bull. Austral. Math. Soc., $22,431-438$.

[11] Beukers, F. (1981) Padé approximations in number theory, Padé approximation and its applications, (Amsterdam, 1980), 90-99, Lecture Notes in Math., Springer, Berlin-New York, 888 .

[12] Beukers, F. (1995) Consequences of Apéry's work on $\zeta(3)$, preprint of talk presented at the Rencontres Arithmétiques de Caen, $\zeta(3)$, Irrationnel: Les Retombées.

[13] Borwein, J. M. \& Bradley, D. M. (1997) Empirically determined Apéry-like formulae for $\zeta(4 n+3)$, Experiment. Math., $6(3), 181-194$.

[14] Borwein, J. M., Broadhurst, D. J. \& Kamnitzer, J. (2001) Central binomial sums, multiple Clausen values, and zeta Values, Experiment. Math., 10, 25-41.

[15] Cohen, H. (1978) Demonstration de l'irrationalite de $\zeta$ (3) (d'aprés R. Apéry), Séminaire de Théorie des Nombres, Grenoble, VI.1-VI.9.

[16] Cohen, J. \& Guy, R. (1997) The Book of Numbers, Copernicus, Springer-Verlag New York, Inc.

[17] Gasper, G. \& Rahman, M. (2004) Basic Hypergeometric Series (Encyclopedia of Mathematics and its Applications), Cambridge Univ. Press, Cambridge.

[18] Gutnik, L. A. (1983) On the irrationality of certain quantities involving $\zeta(3)$, Acta Arith., $42,255-264$.

[19] Jones, W. B. \& Thorn, W. J. (1980) Continued Fractions Analytic Theory and Applications, In: Encyclopedia of Mathematics and its Applications, Addison-Wesley, London. 
[20] Koekoek, R. \& Swarttouw, R. F. (1998) The Askey-scheme of hypergeometric orthogonal polynomials and its $q$-analogue, Report 98-17, Faculty of Technical Mathematics and Informatics, Delft University of Technology.

[21] Nesterenko, Y. V. (1996) A few remarks on $\zeta(3)$, Math. Notes, 59 (6), 625-636.

[22] Nesterenko, Y. V. (2003) Integral identities and constructions of approximations to zeta values, J. Théor. Nombres Bordeaux, 15, 535-550.

[23] Nikiforov, A. F. \& Uvarov, V. B. (1988) Special Functions in Mathematical Physics, Birkhauser Verlag, Basel.

[24] Perron, O. (1910) Über ein Satz des Herrn Poincaré, J. Reine Angew. Math., Über die Poincarésche lineare Differenzgleichung, 137, 6-64.

[25] Petkovsek, M., Wilf, H. S. \& Zeilberger, D. (1997) $A=B$, A. K. Peters, Ltd., Wellesley, M.A.

[26] Pilehrood, H. \& Pilehrood, T. H. (2010) Generating function identities for $\zeta(2 n+2), \zeta(2 n+$ 3) via the WZ method, Electron. J. Combin., 15, 223-236.

[27] Pilehrood, H. \& Pilehrood, T. H. (2009) Series acceleration formulas for beta values, Discrete Math. Theoret. Comput. Sci., 12 (2), 1-9.

[28] Poincaré, H. (1885) Sur les équations linéaires aux différentielles et aux différences finies, Amer. J. Math., 7, 203-258.

[29] Prévost, M. (1996) A new proof of the irrationality of $\zeta(2)$ and $\zeta(3)$ using Padé approximants, J. Comput. Appl. Math., 67, 219-235.

[30] Ram, M. (2008) Transcendental numbers and zeta functions, Math. Student., 77 (4), 45-58.

[31] Raymond, A. (1974) Euler and the zeta function, Amer. Math. Monthly, 81, 1067-1087.

[32] Rivoal, T. (2002) Irrationalité d'au moins un des neuf nombres $\zeta(5), \zeta(7), \ldots, \zeta(21)$, Acta Arith., 103 (2), 157-167.

[33] Soria Lorente, A. (2014) Arithmetic of the values of de Riemann's zeta function in integer arguments, Revista de investigación, G.I.E Pensamiento Matemático, ISSN 2174-0410, IV, 033-0044, 2014.

[34] Soria Lorente, A. (2014) Nesterenko-like rational function, useful to prove the Apéry's theorem, Notes Number Theory Discrete Math., 20 (2), 79-91.

[35] Sorokin, V. N. (1993) Hermite-Padé approximations for Nikishin systems and the irrationality of $\zeta(3)$, Communications of the Moscow Math. Soc., 49, 176-177.

[36] Sorokin, V. N. (1998) Apéry’s theorem, Vestnik Moskov. Univ. Ser. I Mat. Mekh. [Moscow Univ. Math. Bull.], 3, 48-52. 
[37] Spinade, W. (2003) La familia de número metálicos, Cuadernos del CIMBAGE, 6, 17-44.

[38] Takaaki, M. (2014) Negation of the conjecture for odd zeta values, International Journal of Pure and Applied Mathematics, 91 (1), 103-111.

[39] Van Assche, W. (1999) Multiple orthogonal polynomials, irrationality and transcendence, Contemp. Math., 236, 325-342.

[40] Van Assche, W. (2010) Hermite-Padé Rational Approximation to Irrational Numbers, Computational Methods and Function Theory, 10 (2), 585-602.

[41] Van der Poorten, A. (1978/79) A proof that Euler missed... Apéry's proof of the irrationality of $\zeta(3)$, Math. Intelligencer, 1, 195-203.

[42] Zudilin, W. (2001) One of the eight numberss $\zeta(5), \zeta(7), \ldots, \zeta(17), \zeta(19)$ is irrational, Mat. Zametki [Math. Notes], 70 (3), 472-476.

[43] Zudilin, W. (2001) Irrationality of values of zeta function at odd integers, Uspekhi Mat. Nauk [Russian Math. Surveys], 56 (2), 215-216.

[44] Zudilin, W. (2001) One of the four numbers $\zeta(5), \zeta(7), \zeta(9), \zeta(11)$ is irrational, Uspekhi Mat. Nauk [Russian Math. Surveys], 56 (4), 149-150.

[45] Zudilin, W. (2002) A third-order Apéry-like recursion for $\zeta$ (5), Mat. Zametki [Math. Notes], $72(5), 796-800$.

[46] Zudilin, W. (2003) Well-poised hypergeometric service for diophantine problems of zeta values, J. Théor. Nombres Bordeaux, 15 (2), 593-626. 